\title{
Quantum logic as a dynamic logic
}

\author{
Alexandru Baltag • Sonja Smets
}

Received: 19 June 2009 / Accepted: 14 October 2009 / Published online: 12 October 2010

(C) The Author(s) 2010. This article is published with open access at Springerlink.com

\begin{abstract}
We address the old question whether a logical understanding of Quantum Mechanics requires abandoning some of the principles of classical logic. Against Putnam and others (Among whom we may count or not E. W. Beth, depending on how we interpret some of his statements), our answer is a clear "no". Philosophically, our argument is based on combining a formal semantic approach, in the spirit of E. W. Beth's proposal of applying Tarski's semantical methods to the analysis of physical theories, with an empirical-experimental approach to Logic, as advocated by both Beth and Putnam, but understood by us in the view of the operationalrealistic tradition of Jauch and Piron, i.e. as an investigation of "the logic of yes-no experiments" (or "questions"). Technically, we use the recently-developed setting of Quantum Dynamic Logic (Baltag and Smets 2005, 2008) to make explicit the operational meaning of quantum-mechanical concepts in our formal semantics. Based on our recent results (Baltag and Smets 2005), we show that the correct interpretation of quantum-logical connectives is dynamical, rather than purely propositional. We conclude that there is no contradiction between classical logic and (our dynamic reinterpretation of) quantum logic. Moreover, we argue that the Dynamic-Logical perspective leads to a better and deeper understanding of the "non-classicality" of quantum behavior than any perspective based on static Propositional Logic.
\end{abstract}

\footnotetext{
A. Baltag

Computing Laboratory, Oxford University, Oxford, UK

e-mail: Alexandru.Baltag@comlab.ox.ac.uk
}

\section{S. Smets $(\varangle)$}

Department of Philosophy and Department of Artificial Intelligence,

University of Groningen, Groningen, The Netherlands

e-mail: SJLSmets@rug.nl

S. Smets

IEG Oxford University, Oxford, UK 
Keywords Dynamic Quantum Logic - Semantics of Experimental Theories · Empirical Conception of Logic · Logic of Quantum Actions

\section{Introduction}

Does quantum mechanics force us to give up some basic principles of classical propositional logic, such as Distributivity (of Conjunction over Disjunction) or the principle of Bivalence? This question intrigued generations of logicians and philosophers (Birkhoff and von Neumann 1936; Putnam 1968; Dummett 1976; Friedman and Glymour 1972; Bell and Hallett 1982; Butrick 1971; Drieschner 1977; Gardner 1971; Stairs 1983; Jauch and Piron 1970; Hughes 1979) and the issue has still not been fully settled: Bacciagaluppi (2008) provides an in-depth overview from a modern perspective and clears up some of the misconceptions in the early discussion.

In this paper, we argue for a negative answer, using our work (Baltag and Smets 2005, 2008) on formal semantics for a Quantum Dynamic Logic. This is a conceptual paper; there are no essentially new mathematical results (although some easy consequences of our previous results are explicitly stated here for the first time). Instead, our aim is threefold: (1) to provide a philosophical and historical justification for the formal setting presented in Baltag and Smets (2005); (2) to use our results and discussion from Baltag and Smets $(2005,2006 a, 2008)$ in order to advocate a new view on quantum logic, seen as a dynamic logic of non-classical information flow; (3) to apply this new perspective back to our original question above, and argue for a negative answer.

More specifically, we justify the semantic setting in Baltag and Smets (2005) by appealing to E. W. Beth's proposals concerning the logical relevance of empiricalexperimental investigations and the value of Tarskian semantical method for the logical understanding of physical theories. We interpret these proposals in accordance to the operational-experimentalist approach to quantum logic developed by the "Geneva school" led by Jauch and Piron (Jauch 1968; Jauch and Piron 1969, 1970; Piron 1976). According to these authors, a "rational" reconstruction of Quantum Mechanics should be stated in terms of the actual operational meaning of the fundamental quantummechanical concepts and principles, i.e. in terms of outcomes of possible experiments ("answers" to "questions") that could in principle be performed on a given physical system. This approach might be called "operational-realist", to distinguish it from the "pure" operationalism of neo-positivists and instrumentalists. ${ }^{1}$

To make explicit this operational meaning in a formal-semantical way, we use the setting of Quantum Transition Systems introduced in Baltag and Smets (2005). These are dynamic models, obtained by abstracting from Hilbert spaces the main physically-relevant operational features: a physical system can be in a number of possible states; the system comes with a number of possible transition relations between states,

\footnotetext{
1 The difference between this approach and a "pure" operationalism is that the state of a system is not reduced to a list of all the observed results of actual experiments. Instead, it is defined in terms of "potential" or "counterfactual" experiments: what would be observed if a certain experiment was performed? This makes sense only in a non-positivist, realist perspective, in which a physical system exists in a definite "state" even if no experiment is ever actually performed.
} 
relations corresponding to various physical actions; some of these actions are "tests" of physical properties, corresponding to successful yes-no measurements; the properties that can be definitely established by such tests are called testable; the testable properties are closed under dynamic modalities, i.e. the fact that a testable property is established by some "test" (of some possibly different property) is itself a testable property. Hence, rather than adopting the traditional view in quantum logic, we are lead to a dynamic logic, based on a classical (Boolean) propositional logic equipped with action modalities to deal with measurements, unitary evolutions as well as entanglement.

Based on this setting, we argue that the correct interpretation of quantum-logical connectives is in terms of dynamic "test"-modalities, rather than as purely propositional operators. Hence, the axioms of quantum logic should not be understood as alternatives to the classical logical principles, but as extensions of classical logic. To put it in a nutshell, our conclusion is that the laws of quantum logic talk about something else than the ones of classical logic: namely, they talk about a non-classical "logical dynamics" of (quantum) information, rather than about some non-classical alternative theory of "static" information (such as in non-Boolean, non-distributive, partial, paraconsistent or fuzzy logics). Moreover, we argue that only such a dynamiclogic perspective can provide a correct understanding of the logical foundations of Quantum Mechanics: in our view, the "non-classicality" of Quantum Mechanics is entirely due to the "non-classical" features of the flow of information in quantum systems.

The background of our position in this paper is the dynamic turn in Logic, a recent trend in logical studies that has been pursued (mainly, but not exclusively) by the Dutch school in modal logic lead by J. van Benthem, see e.g. van Benthem (1996, in press). The aim of logical dynamics is to capture in one and the same logical system both the results and the triggers for information change. This includes the study of traditional notions of logical consequence, but also the study of other informational processes such as dialogues, observations, measurements, communication and computation. This paradigm has given rise to a family of logical systems known as Dynamic Epistemic Logic (DEL), which forms a fast-growing research field today. One of the main ideas is that various non-classical "propositional" logics (such as intuitionistic logic, Lambek calculus, linear logic, relevance logics, non-monotonic logics, belief revision theories etc.) and logical and epistemological concepts, problems and puzzles (such as the nature of "knowledge" and its relation to justified belief, Fitch's "knowability" paradox, Moore sentences etc.) can be better understood and elucidated if we think of them as being about actions, rather than propositions. According to this view, dynamic logics, with their two-tiered syntax (the "propositional" side and the "action" side), provide the proper setting for reinterpreting such non-classical logics (as action logics) in a way that makes them consistent with classical logic. Our dynamic reinterpretation of quantum logic is thus very much in the spirit of this general "dynamic turn" in Logic.

Even more generally, this view is connected to an older tradition in Computer Science of thinking about information systems in a dynamic manner: a "state" of a system is, in this view, identified only by the actions that can be (successfully) performed on the state. This view is embodied in the various semantic notions of 
"process" that have been proposed in the CS literature: labeled transition systems, automata, coalgebras etc. Our work on quantum dynamic logic (Baltag and Smets 2004, 2005, 2006a,b, 2008) connects this tradition with the completely independent, but similar trend towards "dynamification" in the quantum logic community, trend started in Daniel (1989), Faure et al. (1995) and developed by the "Brussels school" in quantum logic, in a series of papers (Amira et al. 1998; Coecke et al. 2001, 2004; Coecke and Smets 2004; Smets 2001a,b, 2006).

\section{Traditional quantum logic}

In a short paragraph of his Grundlagen der Quantenmechanik (von Neumann 1932), von Neumann introduces the notion of a logical calculus based on the relation between the properties of a physical system and the projections defined on a Hilbert Space. This paragraph and the paper he wrote in 1936 together with Birkhoff, form the birth of quantum logic. Their aim was to discover the logical structure underlying Quantum Mechanics. They write that "based on admittedly heuristic arguments, one can reasonably expect to find a calculus of propositions which is formally indistinguishable from the calculus of linear subspaces with respect to set products, linear sums, and orthogonal complements - and resembles the usual calculus of propositions with respect to and, or, and not" (Birkhoff and von Neumann 1936, p. 1). By focusing on this structure, they argue that Distributivity (of conjunction over disjunction) is the "weakest link" in the algebra of logic.

To explain, let us start by assuming that any physical system is at each instant associated with a "state" in a fixed state space $\Sigma$. Piron (1990) refers for this to Cartan, who in 1920 introduced the notion of an abstract state space. For instance, the state space of a simple point particle can be set as $\Re^{7}$, where the coordinates of a state $(\vec{p}, \vec{q}, t)$ are: the components of the momentum $\vec{p}$, the components of the position $\vec{q}$, and the time $t$. The properties of a system correspond to sets of states. Piron considers for example a point particle, for which the set of states $\left\{(\vec{p}, \vec{q}, t) \mid(\vec{p}, \vec{q}, t) \in \Re^{7}\right.$ and $\left.\vec{p}=\vec{p}_{0}\right\}$ characterizes the following property: "the particle possesses momentum $\vec{p}_{0}$ ".

Indeed, in Classical Physics, every "logical property" of a system $\Sigma$, i.e. every set of states $P \subseteq \Sigma$, can be considered as a physically meaningful property (or "proposition"): if the property $P$ holds (i.e. if the system is in one of the states $s \in P$ ), then this is in principle observable. Given the state space $\Sigma$, the collection of all logical properties $\mathcal{P}(\Sigma)$ forms a complete distributive lattice. The lattice order relation is given by set inclusion and corresponds to the notion of logical consequence, or entailment, $P \Rightarrow Q$ between logical properties. The join is the set union $P \cup Q$, corresponding to the disjunction $P \vee Q$ of logical properties. The meet is the intersection $P \cap Q$, corresponding to the conjunction $P \wedge Q$ of logical properties. The bottom element is the empty set $\emptyset$, corresponding to the always-false proposition $\perp$. And the top element of the lattice is the set $\Sigma$ of all states, corresponding to the always-true proposition $T$. The distributive lattice of logical properties forms a (complete) Boolean algebra if we define for every property a unique complement $\neg P:=\Sigma \backslash P$ in correspondence with 
the notion of classical negation. In other words, the logic of all physical properties of a system in Classical Physics is the usual Boolean logic.

However, the structure of the state space and of the associated lattice of properties is different in Quantum Mechanics. According to the Hilbert space formalism introduced by von Neumann, the "state" of a quantum-physical system is represented by a unitary vector in an infinite-dimensional Hilbert space $H$, or more precisely by the corresponding "ray" (one-dimensional subspace) generated by that vector. So the state space $\Sigma$ is given by the family of all such rays. The fundamental quantum-physical phenomenon is superposition: a superposition of two (or more) states is represented in the Hilbert space formalism by a linear combination of (the unitary vectors representing) the given states. For instance, in the famous example of Schrodinger's cat, if we denote by $|1\rangle$ the state corresponding to "alive", and by $|0\rangle$ the state corresponding to "dead", then before opening the box the cat can well be in some superposed state $\alpha|1\rangle+\beta|0\rangle$ (for some $\alpha, \beta \in C$ such that $|\alpha|^{2}+|\beta|^{2}=1$ ).

The physical significance of superposition is that, if a physical experiment cannot distinguish between (the system being in two, or more) possible states $s_{i}$, then it cannot distinguish between them and any superposed states $\sum_{i} \alpha_{i} s_{i}$. Hence, the properties that can be directly "tested" by experiments must be given only by sets of states that are closed under superpositions: in a Hilbert space $H$, this corresponds to closed linear subspaces of $H$. Such properties that can be tested experimentally are called "experimental propositions", or testable properties.

Let us denote by $\mathcal{L}$ the family of all testable properties of a quantum-physical system. Since closure under superpositions is not a trivial requirement, it is obvious that not every logical property is testable: we have $\mathcal{L} \varsubsetneqq \mathcal{P}(\Sigma)$. Moreover, it follows from the above that, when partially ordered by inclusion (logical entailment), the family of testable properties forms a lattice that is isomorphic to the lattice of all closed linear subspaces of an infinite-dimensional Hilbert space. Since the intersection of a family of closed linear subspaces is a closed linear subspace, the meet $P \wedge Q$ is then given by the intersection $P \cap Q$. Hence, we can identify the "quantum conjunction" of two quantum-testable properties $P$ and $Q$ with the classical conjunction $P \wedge Q$ of properties $P$ and $Q$ (seen as "logical properties"). However, the join, which we denote by $P \sqcup Q$, differs from the union $P \cup Q$, being instead given by the closed linear subspace generated by the union. So, "quantum disjunction" $P \sqcup Q$ is weaker than classical disjunction $P \vee Q$. In fact, quantum disjunction captures the physical notion of "superposition": a state satisfies $P \sqcup Q$ iff it is a superposition (linear combination) of states satisfying $P$ or $Q$ (or both). Like $\mathcal{P}(\Sigma), \mathcal{L}$ is also a complete lattice, but it is a non-distributive one: the operation of taking the linear closure of the union does not distribute over intersection.

The complement $\Sigma \backslash P$ of a closed linear subspace is not necessarily a closed linear subspace. Hence, the family $\mathcal{L}$ of testable properties is not closed under classical negation. However, for every testable $P$ there exists a special testable property $\sim P$, which is stronger than classical negation $\neg P$ and is called the ortho-complement of $P$ : in a Hilbert space, it corresponds to the so-called orthogonal complement $P^{\perp}$ of the subspace $P$, composed of all vectors that are orthogonal on every vector in $P$. Note that the orthocomplement $\sim$ satisfies (but is not uniquely determined by) the two usual "Boolean laws" in the lattice $\mathcal{L}: P \wedge \sim P=\perp, P \sqcup \sim P=\top$. In addition, the 
orthocomplement satisfies an interesting law, called the Orthomodular Law: For all $P, Q \in \mathcal{L}, P \subseteq Q$ implies $Q=P \sqcup(Q \cap \sim P)$. This law can be seen as a weakening of the Distributivity laws.

What is traditionally known as "Quantum Logic" is essentially an algebraic investigation of the orthomodular lattice $(\mathcal{L}, \subseteq, \wedge, \sqcup, \sim)$ of all testable properties of a quantum system. Formally, the syntax of Quantum Logic $(Q L)$ is build up from a set of basic formulas $p$ (denoting some basic testable properties):

$$
\varphi::=\perp|p| \sim \varphi \mid \varphi \wedge \varphi
$$

where the "top" and the join are definable in the usual way: $\top:=\sim \perp, \varphi \sqcup \psi:=$ $\sim(\sim \varphi \wedge \sim \psi)$.

At the time when Quantum Logic was born, "formal logic" was conceived as being about providing a purely symbolic calculus, i.e. a syntax together with an axiomatic theory, so Birkhoff and von Neumann did not explicitly give a formal semantics for quantum logic. However, they clearly pointed to the Hilbert space interpretation (in terms of closed linear subspaces). But one of the aims of the early quantum logic project was to provide an axiomatization of Quantum Logic that is both complete (with respect to the Hilbert space interpretation) and given in purely qualitative (algebraic-logical) terms, without any reference to Hilbert spaces, probabilities or other quantitative notions. So the proposal of Birkhoff and von Neumann was that the language of orthocomplemented lattices was the appropriate qualitative abstraction to answer this question.

One problem encountered in the efforts towards a complete axiomatization was the lack of a "quantum implication". Indeed, it seems to be impossible to find an experiment to test a proposition of the form "if $P$ then $Q$ ". ${ }^{2}$ Indeed, there doesn't exist any operation on testable properties that satisfies the classical form of Deduction Theorem for quantum logic. Nevertheless, the above syntax can define a connective $P \stackrel{S}{\rightarrow} Q$, the so-called "Sasaki hook", satisfying a weak property of implication, namely that

$$
P \stackrel{S}{\rightarrow} Q=\top \text { iff } P \subseteq Q
$$

The desired connective is not given by the analogue of material implication $\sim \varphi \sqcup \psi$, but is defined by: $\varphi \stackrel{S}{\rightarrow} \psi:=\sim \varphi \sqcup(\varphi \wedge \psi)$.

To obtain a completeness result, one may first want to have a more abstract formal semantics given in more qualitative terms than the "concrete" Hilbert semantics informally given by Birkhoff and von Neumann. Then Hilbert-completeness could be achieved via a Representation Theorem, stating that every abstract model can be canonically embedded in a "concrete" (Hilbert-space) model. Such an abstract semantical setting, together a modal-logic axiomatization, was proposed by Goldblatt (1984),

\footnotetext{
2 This point was already noted by Birkhoff and von Neumann who remark in one of their footnotes that: "If $a, b$ are two physical qualities, then $a \cup b, a \cap b$ and $a^{\prime} \ldots$ are physical qualities too ... But $a \rightarrow b$ is not a physical quality; it is a relation between physical qualities" (Birkhoff and von Neumann 1936, p. 25).
} 
in terms of orthomodels (Kripke models with a symmetric irreflexive "orthogonality" relation), but it proved not to be Hilbert-complete. Indeed, the (Hilbert-valid) Orthomodularity Law not only fails to be valid on orthoframes, but it corresponds to no first-order frame condition (Goldblatt 1984). ${ }^{3}$ Nevertheless, Piron $(1964,1976)$ proposed an algebraic axiomatization, for which he proved a Representation Theorem w.r.t. "generalized Hilbert spaces". This result was improved by Solèr (1995) and Mayet (1998), who added another axiom, obtaining an (abstract) Hilbert-completeness result (with respect to the standard infinite-dimensional Hilbert spaces). However, this axiomatization is not first-order: some of the axioms cannot be stated in the firstorder (or modal) language of orthomodular lattices, but require quantification over higher-level objects, such as automorphisms of the given lattice. Moreover, some of the axioms have an artificial, un-intuitive character.

\section{On the semantics of experimental theories}

\subsection{Putnam's question: Is logic empirical?}

In 1968, Putnam (Putnam 1968) asked the question with which we started our paper, stating it in the following form: could some of the "necessary truths" of classical logic ever turn out to be false for empirical reasons? Putnam gave a positive answer to this question, based on what we called the "traditional" view of Quantum Logic, and used it in support of his argument for a realist interpretation of quantum mechanics. As we saw, this "traditional" view is that Quantum Logic is an alternative propositional logic, having different laws than classical logic (e.g. Distributivity is replaced by a weaker version, Orthomodularity), but based on the same basic connectives and acting at the same (propositional) level as classical logic. As a consequence, Putnam argued that adopting Quantum Logic implies a revision of classical logical principles. Not surprisingly, his 1968 paper triggered a lively debate among philosophers, physicists and quantum logicians about the nature of quantum logic and the very idea of having to revise our classical logic. There are many stances in this debate (see e.g. Dummett 1976; Friedman and Glymour 1972; Bell and Hallett 1982; Butrick 1971; Drieschner 1977; Gardner 1971; Stairs 1983; Jauch and Piron 1970; Hughes 1979).

According to an excellent modern overview of the discussion (Bacciagaluppi 2008), the currently most widely-held view seems to be based on the following three points: (a) quantum logic is suitable as a logic that locally replaces classical logic when used to describe "a class of propositions in the context of quantum mechanical experiments", (b) it is still open whether or not quantum logic does globally replace classical logic, (c) quantum logic does not solve the quantum mechanical paradoxes.

The last of these points is probably the least controversial one and hence we will not give it much further attention in this paper. With respect to point (a), we have no problems if it is understood as saying that quantum logic is fit to describe quantum testable properties. However, not all physically meaningful propositions that can be

\footnotetext{
3 But note that the axioms of an orthomodular lattice are also Hilbert-incomplete (see Dalla Chiara et al. 2004 for a counter-example).
} 
uttered about quantum systems deal with properties that are directly testable (in the sense of corresponding to closed linear subspaces of a Hilbert space).

Examples of properties that are not "testable" according to Quantum Logic are:

- "The System is not in state $s "$

- "The system is in state $s$ or state $t$ "

- "The system is in a proper superposition state"

- "The system is in an entangled state"

- "The system is in a separable state" etc.

None of these properties is closed under superpositions (linear combinations). For instance, a linear combination of entangled states can be separable (i.e. nonentangled!), and vice-versa. However, it is obvious that properties like "entanglement" and "separability" are not only physically meaningful, but indispensable for any formulation of Quantum Mechanics. Such "not-testable" properties may not be directly tested by an experiment, but they can be inferred indirectly by testing other, stronger properties. For instance one can find out that the system is actually in some other state $t \neq s$ (by performing a successful test of state $t$ ), and thus infer that it is not in state $s$. Thus, "non-testable" does not imply "physically meaningless". Hence even within quantum logic there should be space for classical logic. Moreover, even if one agrees that "quantum-testable" properties form a class that is of intrinsic interest, this still leaves the question of why these particular type of properties obey different logical laws. Simply deciding in an ad-hoc manner to "locally replace" classical logic when dealing with some specific domain of application cannot by itself constitute a principled answer to the above question!

In other words, even if one concedes that in some sense logic is "empirical" and that the classical laws could in principle fail, or be unapplicable to some given class of relevant propositions, it still needs to be checked if these laws actually do fail; and, if so, the fact still needs to be explained: in what sense these laws fail, and why? Only this would make intelligible and rational the otherwise apparently mystical action of "locally replacing" classical logic with another one.

\subsection{Beth's views on semantics and empirical sciences}

The whole debate is still very interesting today, but what is maybe less well known is that Putnam was not the first to wonder about these issues. The view that classical logical laws are under threat due to the results of empirical investigations in the quantum realm, traces back to the work of the Dutch logician E.W. Beth.

Indeed, the development of quantum logic in the early thirties also caught Beth's attention. ${ }^{4}$ At a symposium in Scheveningen in September 1947, Beth explains that von Neumann and others concluded that adopting the quantum principle of comple-

\footnotetext{
4 Among the first publications of Beth on this topic we find (Beth 1949a) as well as a summary of one of his lectures held in Paris during the workshop "Les Journées d'études de logique symbolique de Paris" organized by J.-L. Destouches at the Institut Henri Poincaré in January 1948. Beth gave two lectures at this workshop in Paris, in the last one he presented his work on the application of semantics to theories of physics (Beth 1948/49).
} 
mentarity boils down to a weakening of our logic. This led in his view to a real crisis of certainties, a change of our logical laws which is due not to a priori insight but due to the results of empirical investigations (Beth 1948). After Beth ended his presentation in Scheveningen, he got questioned on exactly this point. In his replies (which were published in Dutch in (Beth 1948)) he argued that in order to explain "phenomena of light" we need to look for a new language with new semantic and syntactic laws. Every language should be adapted to the structure of reality that is to be described. For Beth, a change of logic was a plausible option, as by weakening certain logical laws it becomes possible to reconcile contradictory theories. At the end of the 1940s, Beth consistently defended the relevance of empirical-experimental science to Logic, and argued that in particular quantum theory seems to require an abandonment of some of the classical logical principles. Indeed, in his 1948 text "Science as a cultural factor" (Beth 1948), Beth writes: "The principle of complementarity, formulated by Bohr, eliminated these embarrassing inferences from both theories [corpuscular and undular theories of light] with one stroke. On closer investigation von Neumann (1932) and others concluded that acceptance of the complementarity principle amounts to a weakening of logic. (...) This a veritable 'foundational crisis'. Until recently, a radical change in the established laws of logic, not by virtue of aprioristic insight, but as a consequence of certain results of experimental research, was the very last thing that was considered possible. So it could have been expected that many would protest against this development in physics. Humanly speaking, these protests are understandable, but they lack objective foundation. (...) It will now be evident that the foundational crisis in the exact sciences - the example discussed above is only one of many cases - in no sense justifies misgivings as to the future of science. On the contrary: abandonment of principles once considered unassailable is the price to be paid for the progress of science" (Beth 1948, pp. 5-6).

This seems a clear defense of the same point of view later exposed by Putnam: Logic, as a discipline within Philosophy of Science, must "give in" to Physics; the applicability of logical laws to specific areas of reality is an empirical problem, which must be decided by empirical science. But on the other hand, in another 1948 text, Beth advocates a much more "active" attitude for philosophical logicians towards empirical science: "I would also like to emphasize that scientific philosophy does not necessarily have to take up a purely passive attitude towards science. On the contrary, it may influence the development of science by stimulating research and by exposing pseudo-scientific tendencies" (Beth 1968a, p. 31).

From deferential acceptance to "exposure" of pseudo-scientific "lessons" that some may hurry to extract from the latest scientific breakthroughs: the job of the "scientific philosopher" suddenly becomes rather exciting! Moreover, on the same page Beth explains that part of this job is to be taken by the logician: "The most important methods applied in scientific philosophy are: (1) Logical analysis, as propagated by Bertrand Russell and by the Vienna Circle. (2) Significs, socio-psycho-linguistic or semiotic procedures (Mannoury, C.W. Morris, Hollitscher). (3) Historical research (Couturat, Duhem, Enriques, Kelsen)" (Beth 1968a, p. 31). In the next paragraphs, Beth relates this task to the use of Tarski's semantic method, giving it as an example of the successful use of Logic in dispelling the "pseudo-scientific tendencies" inspired in some philosophers by the existence of classical paradoxes: "The power of 
the method of logical analysis is perhaps best demonstrated by Tarski's investigations into the Aristotelian or absolute notion of truth. The Ancients already observed that the unlimited application of this notion gives rise to a contradiction which is known as the Paradox of the Liar. Modern logic has given this paradox a form which shows that it cannot be dismissed as a mere fallacy. Tarski has established a definition of truth which does justice to the intention of Aristotle's definition without leading to contradictions" (Beth 1968a, p. 31). In the same text, on page 33, Beth goes on to argue for the value of Tarski's semantical method in the analysis of scientific theories: "Semantics provides us with a "doctrine des vérités préalables" as postulated by Gonseth. I think it is a pity that Gonseth does not so far accept the methods and the results of semantics. Only a few months ago (Gonseth 1948) he repeated two objections which he had already made several years before (Gonseth, 1938, 1939) and which, in his opinion, had not been answered, namely: (1) semantics is based on naive realism, and (2) it is intended to give once for all a basis for all scientific activity. As a matter of fact, the first objection has been dealt with by Tarski himself (1944), while the second betrays a complete misunderstanding of the aims, the methods and the results of semantics. Therefore I would like to answer both objections together by pointing out that semantics in no way adopts a dogmatic point of view. It aims at clarifying the foundations of science, not at establishing an unshakable conviction. It achieves this by laying bare the hidden assumptions underlying symbolic logic and formal axiomatics. It does not claim to be able to justify these assumptions, but only shows that modern science takes them for granted."

At the time, the semantical method was new and still debatable even within modern (formal) Logic. The standard way to present a logic was in purely syntactic-axiomatic terms, as a symbolic calculus, with no need for a formal semantics. Indeed, in a much later text, Beth writes: "Traditional logic operated on a semantical plane, i.e. in investigating the demonstrative force of the arguments it started from the meanings of the words and symbols occurring in those arguments. At first modern logic employed a purely formalistic method, which left these meanings out of account. Until Tarski gave a proof to the contrary, it was even believed that a semantic approach could never be exact" (Beth 1968b, p. 101).

Van Fraassen underlines the novelty of Beth's semantical approach to philosophy of scientific theories: "But the most remarkable feature of the book [Natuurphilosophie], in retrospect, is an about-turn that appears in a note added to the book just before publication (p. 133). The change is a shift from rules of deduction still reminiscent to Carnap's 'logical syntax' to semantic consequence relations in the sense of Tarski. It may not have seemed a great departure to Beth at the time, but in fact it is a turning point; to my mind it is the birth of what came to be called the semantic approach or semantic view later on. The new way of looking at physical theory here indicated is not developed in the book, but introduced in two short articles published around the same time (1948, 'Analyse sémantique des théories physiques'; 1949, 'Towards and up-to-date philosophy of the natural sciences') and repeated - equally briefly-in the 1960 paper 'Semantics of physical theories'. Reading the book with this ending in mind, the path to this break is visible from the beginning, and seems inevitable given how Beth viewed the historical development of the physical sciences" (van Benthem et al. 2008, p. 22). Van Fraassen summarizes Beth's views and their contemporary 
significance as follows: "In his work in philosophy of science, E.W. Beth's aim was to apply the methods of formal semantics (as developed by Tarski et al.) to the analysis of theories in the natural sciences. (...) Beth then developed this point of view, which is related to those of von Neumann, Birkhoff, Destouches, and Weyl, in three articles (Beth 1948/49, 1949b, 1960). As we shall suggest, it is a point of view which has close affinities to much contemporary foundational work in physics. Beth's opinion, in which I concur, is that his semantic approach represents a much more deep-going analysis of the structure of physical theories than the axiomatic and syntactical analysis which depicts such a theory as a symbolic calculus interpreted (partially) by a set of correspondence rules" (van Fraassen 1970, p. 325). As van Fraassen (1970, 1987) explains, the main idea is to exchange the axiomatic theory for models representing the actual behavior of a physical system. In this semantic account one starts with a set of states and specifies when a proposition (concerning physical magnitudes taking a certain value at a certain time) is true. Overall, this provides us with a characterization of physical theories which is "more faithful to current practice in foundational research in the sciences than the familiar picture of a partly interpreted axiomatic theory" (van Fraassen 1970).

\subsection{The operational approach of the Geneva school}

The so-called "operational approach" to quantum logic originated in the work of Jauch and Piron (Jauch 1968; Jauch and Piron 1969; Piron 1976). One of the underlying aims of their approach was to construct a theory about quantum systems that stays as close as possible to the phenomena, introducing theoretical concepts only when they are directly and convincingly motivated by empirical facts (Jauch and Piron 1970). Jauch and Piron refer to Birkhoff and von Neumann's (1936) as a point of departure for what they call "the axiomatic reconstruction of quantum mechanics". However, they find very unfortunate the use of the word "logic" in the title of Birkhoff and von Neumann's paper: "Claims have been made that the paradoxes in quantum mechanics disappear only if they are analyzed not with ordinary logic but with a new kind of quantum logic instead. It should perhaps be mentioned that Bohr, on several occasions, rejected this point of view and Pauli too, has expressed similar doubts" (Jauch and Piron 1970, p. 167). Similarly, they note that the attempt of interpreting their own empiricallydriven calculus for micro-systems as a non-classical logic, leads to difficulties. Their calculus is build up from propositions that are based on so-called yes-no experiments, or "questions." The already mentioned impossibility of finding experimental arrangements to measure propositions of the form "if $P$ then $Q$ " is seen by Jauch and Piron as indicating that "it would thus be better to avoid the term logic altogether in the

\footnotetext{
5 A "yes-no question" or so-called "definite experimental project" is taken "to be a real experimental procedure where we have defined in advance what would be the positive response should we perform the experiment" (Moore 1999, p. 65). We can think of the experimental procedure as a list of concrete actions which explicitly must or must not be performed in a specific way. Given that we execute such a procedure and that the conditions defining a positive result are known, we assign the response "yes" to the question if the conditions are satisfied and the response "no" otherwise. To summarize we quote Cattaneo and Nisticó (1991): "A question is both: (1) a description of an experiment to be carried out on the physical system considered; (2) a rule enabling us to interpret the possible results in terms of "yes" or "no"” (p. 1298).
} 
designation of the lattice structure of general quantum mechanics" (Jauch and Piron 1970, p. 180).

Abandoning the name "logic" in Quantum Logic, as Jauch and Piron suggested, depends on what one understands "logic" to be about. Jauch and Piron adopted a clear operational and experimentalist point of view, a view which ties in close to our own framework of quantum logical dynamics and action-based reasoning. What they witnessed was the failure of describing static logical constructs via experimental means. Hence from our perspective, their conclusion can better be rephrased as follows. There is no problem with the term "logic" in the designation of the lattice structure of general quantum mechanics as long as this term is not restricted to static propositional logic. In our view the term "logic" incorporates "logical dynamics" and as such it does apply well to Quantum Logic. But the Jauch-Piron reluctance to use the term "logic" points precisely to the fact that this structure should not be understood as being on the same level as classical propositional logic.

\section{Dynamic quantum logic}

We have seen that, in accordance to the traditional view on Quantum Logic, the "logic" of quantum-testable properties is rather strange. Instead of a Boolean algebra, we get a non-distributive, orthomodular lattice, endowed with a negation-like orthocomplement operation. But does this imply that Quantum Mechanics requires us to give up the classical logical laws governing "static" properties or "propositions"? In this section we will argue that this is not the case.

Our goal is to follow Beth and the operationalist approach by first looking carefully at the actual physical and operational meaning of quantum-logical connectives. What we discover is that this meaning is essentially dynamic, having to do with the possible outcomes of "actions" (measurements) that can be performed on a system. Then we combine this operational perspective with Beth's insistence on the value of a formal semantical approach to physical theories: we give a formal semantics for a dynamic logic, in the standard style used nowadays in Computer Science and Modal Logic (using "transition systems", a model for dynamics essentially based on Kripke's semantics for modal logic, itself an adaptation of Tarski's semantics for firstorder logic). We present a few results, then present our conclusions concerning the philosophical discussion presented in the previous sections.

\subsection{Operational meanings are dynamical}

Let us go back to the original "concrete" semantics for quantum logic, in terms of an infinite-dimensional Hilbert space. Why are "testable properties" to be identified with closed linear subspaces of $H$ ? Previously, we answered this question by saying that experiments cannot distinguish between states and their superpositions. But let us look more closely at the notion of "experiment" or "test".

The prototypical quantum-mechanical experiment is given by measurements. A "measurement" is represented in the Hilbert-space formalism by a family of projectors onto mutually orthogonal subspaces of $H$. In principle, every measurement can 
be reduced to a combination binary measurements, also called bit measurements. A binary measurement is a pair of projectors, one onto some give some linear subspace $P$, and the other onto its orthogonal complement, denoted by $P^{\perp}$ or $\sim P$. Following Piron, we can think of a binary measurement as a yes/no question; if the answer is "yes", then property $P$ is established: it is known that $P$ holds after the measurement (at the output-state); if the answer is "no", then $\sim P$ holds after the measurement (at the output-state). We will denote the first projector by $P$ ? and call it "(successful) test $t^{6}$ of property $P$ "; the second projector will be denoted by $(\sim P)$ ?, and call it "failed (unsuccessful) test of $P$ " or equivalently "(successful) test of $\sim P$ ".

The important thing is that performing a successful test $P$ ? only establishes that $P$ holds after the test. A quantum measurement typically changes the original state of the system: if the initial state is $s$, then Quantum Mechanics tells us that the state after a successful test $P$ ? is given by the result of projecting $s$ onto the subspace $P$, i.e. by applying the projector to the original state to obtain $P$ ? $(s)$ as the new state. This new state $P$ ? $(s)$ lies indeed within the subspace $P$. But nothing guarantees that the original state $s$ satisfied $P$ as well!

Moreover, the actual (yes/no) outcome of a measurement is not always uniquely determined by the input-state. Quantum measurements are inherently nondeterministic; if the input-state $s$ did not belong to subspace $P$ nor to subspace $P^{\perp}$, then both answers (yes and no) may be possible (with some probabilities). So even if we somehow knew the original state $s$ of the system, we may still be unable to predict with certainty the result of the measurement (except in very special cases). However despite being non-deterministic, quantum measurements have the property that they are in a sense "consistent". If a "yes" or "no" answer has been obtained after a measurement of $P$, thus "establishing" property $P$, then the same answer will be obtained (with probability 1 ) if the same measurement is immediately repeated. As a consequence, quantum tests (projectors) are said to be idempotent (where ; represents the composition of tests):

$$
P ? ; P ?=P \text { ? }
$$

More generally, a measurement of $P$ gives the "yes" answer with probability 1 if and only if the state $s$ of the system before the measurement lied within the space $P$ (i.e. $s \in P$ ). This is the reason for which Jauch and Piron identify Einstein's "elements of reality" (the "real" properties that actually hold in a system in state $s$ ) with the questions that are guaranteed to received the answer "yes" (when performed on a system in state $s$ ): the tests that are necessarily successful (when the initial state $s$ ). These correspond precisely to the closed linear subspaces $P$ such that $s \in P$. Hence, a "testable property" can be identified with a closed linear subspace. A testable property $P$ holds iff the test $P$ ? of $P$ will surely succeed.

What is then the operational meaning of the orthocomplement operation $\sim P$ ? Suppose that for a measurement, we identified one of the possible outcomes as the "yes" answer, the other one as the "no" answer, and this defined a successful test $P$ ?, and

\footnotetext{
6 These tests correspond to what has been called "filters" by Piron (1976). A filter is an "ideal measurement of the first kind" (in the sense of W. Pauli).
} 
thus a testable property represented by the subspace $P$. We can now consider the same measurement, but in which the "yes" and "no" answer have been interchanged: this is another "question". What is the testable property associated to the successful test corresponding to this question? Obtaining the answer "yes" to this new question with probability 1 is the same thing as obtaining the answer "no" to the original question with probability 1 . In other words, this new test is guaranteed to succeed if and only if the original test is guaranteed to fail.

This is the true meaning of orthocomplement: $\sim P$ holds (at a state $s$ ) iff the test $P$ ? for $P$ will surely fail (when performed on state $s$ ). This is not the same as saying that $P$ is false at $s$, but is much stronger: as already observed, $P$ may have been false at $s$ (i.e. $s \notin P$ ), and still there might be a non-zero probability that the test $P$ ? will succeed (when $s$ is neither in $P$ nor in the orthogonal subspace $P^{\perp}$ ).

We can easily capture the meaning of orthocomplement in a formula if we use the Dynamic Logic notation. Recall that, for an "action" or "program" $\pi$ and a property $P$, one can define in Dynamic Logic a new property $[\pi] P$ meaning that: if action $\pi$ was performed (on the current state of the system), then after that property $P$ would surely hold (at the output-state). The operator $[\pi]$ is called a dynamic modality, and the property $[\pi] P$ is called the "weakest precondition" that ensures $P$ (will necessarily hold) after (performing) $\pi$. The operational meaning of orthocomplement can be now formally stated as

$$
\sim P=[P ?] \perp
$$

(where $\perp$ is the "always false" proposition): i.e. a successful test of $P$ is impossible (on the current state). The law of double-orthocomplementation $\sim P=P$ can also be justified in this way: since $\sim \sim P$ is by definition the same as $[(\sim P)$ ?] $\perp$, this property holds iff a successful test for $\sim P$ is impossible; but as we saw, a successful test for $\sim P$ is nothing but an unsuccessful test for $P$; hence, $\sim \sim P$ holds iff an unsuccessful test for $P$ is impossible, i.e. iff the test for $P$ will surely succeed, which is the same as saying that $P$ itself holds.

What is the operational meaning of conjunction $P \wedge Q$ ? In other words, why does the classical conjunction (intersection) of two testable properties give us a testable property? The reason is that experiments are closed under non-deterministic choice. Given two tests $P$ ? and $Q$ ?, we can design a new experiment, consisting of: first choosing arbitrarily either one of the two tests ( $P$ ? or $Q$ ?); then performing the chosen test, and recording the answer. It is easy to see that this new experiment will surely succeed (i.e. regardless of the choice) if and only if performing any of the two tests (on the curely) will surely succeed. In other words, the testable property tested by this experiment holds iff both properties $P$ and $Q$ hold. Hence, this testable property must correspond to the intersection $P \wedge Q$.

This clever argument, conceived by Piron, can also be formalized in Dynamic Logic. Recall that in Dynamic Logic (as elsewhere in Computer Science), the arbitrary (non-deterministic) choice between two actions $\pi_{1}$ and $\pi_{2}$ ("do either $\pi_{1}$ or $\pi_{2}$ ") is denoted by $\pi \cup \pi_{2}$. Recall also the standard Dynamic Logic identity $\left[\pi_{1} \cup \pi_{2}\right] P=$ $\left[\pi_{1}\right] P \wedge\left[\pi_{2}\right] P$, saying that a property is guaranteed to hold after performing nondeterministically either $\pi_{1}$ or $\pi_{2}$ (chosen arbitrarily) iff it is guaranteed to hold both 
after performing $\pi_{1}$ and after performing $\pi_{2}$. But, since the dynamic modalities can only directly express (as $[\pi] \perp$ ) the necessary failure of an action (but not necessary success!), we need to go via the orthocomplements when designing the above experiment: first choose arbitrarily either one of the two tests ( $P$ ? or $Q$ ?); then performing the chosen test, but with the "yes" and "no" answers interchanged. This experiment can be denoted by $(\sim P)$ ? $\cup(\sim Q)$ ?. Such an experiment will necessarily fail if and only if both $P$ and $Q$ hold, as witnessed by the following piece of equational reasoning:

$$
[(\sim P) ? \cup(\sim Q) ?] \perp=[(\sim P) ?] \perp \wedge[(\sim Q) ?] \perp=\sim \sim P \wedge \sim \sim Q=P \wedge Q
$$

A similar argument can be given for the testability of quantum join $P \sqcup Q$, though this of course follows from the above together with the identity $P \sqcup Q=\sim(\sim P \wedge \sim Q)$. Finally, the operational meaning of the "Sasaki hook" (quantum implication) $P \stackrel{S}{\rightarrow} Q$ is surprisingly simple: $P \stackrel{S}{\rightarrow} Q$ holds iff, after successfully performing $P$ ? (on the current state of the system), property $Q$ will surely hold (at the output-state, with probability 1). In other words, "quantum implication" is not a "static" implication at all, but it's simply the "weakest precondition" captured by the dynamic modality for tests:

$$
P \stackrel{S}{\rightarrow} Q=[P ?] Q
$$

\subsection{The logic of quantum actions}

In our technical papers (Baltag and Smets 2005, 2006a, 2008), we presented models for a Quantum Dynamic Logic, that encode the above-described "operational" meanings into a formal semantics, in accordance with Beth's requests. We briefly summarize here these technical results.

In Baltag and Smets (2005) we gave an abstract notion of relational (Kripke) models for quantum logic, models called Quantum Transition Systems (QTS). A QTS $\Sigma=(\Sigma, \stackrel{P}{\rightarrow}, \stackrel{a}{\rightarrow})_{P \subseteq \Sigma, a \in \mathcal{A}}$ consists of a set of states $\Sigma$ and a family of transition relations (binary accessibility relations) between those states, relations labeled either by subsets $P \subseteq \Sigma$ of the state space or by elements $a$ of a set $\mathcal{A}$ of basic "actions". As before, states represent possible states of a physical system; and the transition relations describe the changes of state induced by possible actions that may be performed on the system. The relations of the form $\stackrel{P \text { ? }}{\rightarrow}$ correspond to "quantum tests" of the (strongest testable property implied by) property $P$, while the relations of the form $\stackrel{a}{\rightarrow}$ correspond to "unitary actions" (also called "reversible evolutions" in Quantum Mechanics, and "quantum gates" in Quantum Computation). A QTS is required to satisfy a set of ten semantical frame conditions, that encode natural properties of measurements and evolutions.

An example of QTS is the "concrete" QTS $\Sigma(H)$ given by an infinite-dimensional Hilbert space $H$ : here, the "states" correspond to rays (one-dimensional subspaces), while basic transition relations correspond to special kinds of linear maps on $H$ : the 
tests $\stackrel{P}{\rightarrow}$ are partially functional relations, given by the projector maps onto the closed linear subspace $\bar{P}$ generated (by the set of states corresponding to) property $P$; the "unitary actions" are unitary linear maps $U$ on $H$.

In Baltag and Smets (2005) we prove a Representation Theorem, saying that every ("abstract") QTS is representable as such a "concrete" QTS, i.e. it can be canonically embedded in the QTS associated to an infinite-dimensional Hilbert space. Thus, our work improves on the older representation results by Piron, Solèr, Mayet and others on the Hilbert-complete axiomatizations of algebraic quantum logic. As already mentioned in the previous section, the Solèr-Mayet axiomatization was not firstorder (in the language of orthomodular lattices), but it quantified over higher-order notions (such as the lattice automorphisms). Our dynamic-logical perspective treats "actions" seriously, as first-class objects of the logic (instead of refering to them indirectly as second-order concepts). This allows us to encode higher-order aspects into a modal language (with action modalities). So while our axiomatization is clearly different from the earlier results of Piron, Solèr and Mayet, the proof of our Hilbertcompleteness result does connect back to theirs. For further details on these aspects we refer the interested reader to Baltag and Smets (2005).

In any $Q T S$, one can generate a larger family of "quantum programs" $\pi$ by closing the family of basic transition relations under non-deterministic choice $\pi_{1} \cup \pi_{2}$ (given by the union of the two relations, seen as sets of pairs), and relational composition $\pi_{1} ; \pi_{2}$. The first operation $\pi_{1} \cup \pi_{2}$ expresses non-determinism: "do either $\pi_{1}$ or $\pi_{2}$ "; the second operation $\pi_{1} ; \pi_{2}$ expresses temporal succession of actions: "first do $\pi_{1}$ then do $\pi_{2}$ ". One may also consider iteration (Kleene star) of action $\pi^{*}$, but this is not needed for our purposes.

The language of our logic is taken to be the same language as the star-free fragment of Propositional Dynamic Logic (PDL). PDL has been developed in the domain of computer science, and is fit to reason about and verify the properties of programs (Harel et al. 2000). The syntax of $P D L$ consists of two levels. The upper level is said to be "static" and consists of propositional formulas. The lower level is taken to be "dynamic" and consists of actions. Both levels are defined by mutual induction:

$$
\begin{aligned}
& \varphi::=c|p| \neg \varphi|\varphi \wedge \varphi|[\pi] \varphi \\
& \pi::=a|\varphi ?| \pi \cup \pi \mid \pi ; \pi
\end{aligned}
$$

where the $c$ 's come from a set of basic propositional constants that includes an "always false" constant $\perp$, the variables $p$ range over a given set of basic atomic propositions, and the basic action labels $a$ come from the same set $\mathcal{A}$ as the ones in our models. As usual, $\neg$ is classical negation, $\wedge$ is classical conjunction. The semantics is given in a QTS in the obvious way, mapping each sentence $\varphi$ to a set $\|\varphi\| \subseteq \Sigma$ of states satisfying it, and mapping each program $\pi$ to a transition relation $\stackrel{\pi}{\rightarrow} \subseteq \Sigma \times \Sigma$ : tests $\varphi$ ? are interpreted using the transition relations labeled by $\|\varphi\|$ ? etc; the dynamic modality is interpreted in the usual way, using the weakest precondition: $\|[\pi] \varphi\|=\{s \in$ $\Sigma: \forall t(s \stackrel{\pi}{\rightarrow} t \Rightarrow t \in\|\varphi\|)\}$. All other propositional operators keep their Boolean interpretation. 
The resulting logic is called the Logic of Quantum Actions ( $L Q A)$ in Baltag and Smets (2005). The reason we allow classical negation is the one explained in the previous section: since testable properties are not the only physically meaningful properties, we want to allow our logic to express as many such physical properties as possible. Indeed, not only we obtain a dynamic interpretation of all the non-classical connectives of traditional Quantum Logic, but (due to the presence of classical negation $\neg$ in $L Q A$ ) $L Q A$ has strictly more expressive power than Quantum Logic. We can take e.g. the classical dual $\langle\varphi ?\rangle \psi:=\neg[\varphi$ ?] $\neg \psi$ of the weakest precondition, which corresponds to the existential modality in $P D L$ : this expresses the possibility of actualizing a property $\psi$ by a successful test of property $\varphi .^{7}$ This allows us to express that a property $\varphi$ is potentially true at a given state, i.e. that it can be actualized by some measurement performed on the current state. Or equivalently we can say that the test $\varphi$ ? may be successful. So the formula $\diamond \varphi$ expressing potentiality of $\varphi$ can be defined as the possibility of testing for $\varphi$, i.e. $\nabla \varphi:=\langle\varphi$ ? $\rangle$.

It is important to note that (even if we eliminate classical negation) $L Q A$ is a bivalent logical calculus, having only two truth values. Hence propositions in this logic either hold at a given state or they don't, there are no extra values or value gaps in $L Q A$.

Traditional quantum logic $Q L$ can be re-interpreted inside $L Q A$, by defining inductively a translation map, that maps every formula $\varphi$ of $Q L$ into a formula $\varphi^{\text {tr }}$ of $L Q A$ : $\perp^{t r}=\perp, p^{t r}=p,(\sim \varphi)^{t r}=\left[\varphi^{t r} ?\right] \perp,(\varphi \wedge \psi)^{t r}=\varphi^{t r} \wedge \psi^{t r}$. It is easy to see that the translation map is faithful on Hilbert spaces: if $\Sigma(H)$ is the concrete QTS induced by a Hilbert space $H$, then for all states $s$ and all $Q L$-formulas $\varphi$ we have

$$
s \models_{H} \varphi \text { iff } s \models \Sigma(H) \varphi^{t r} .
$$

Moreover, one can see that Quantum Logic is in fact equivalent (co-expressive) on Hilbert spaces with the negation-free unitary-action-free fragment of $L Q A$ : every formula of $L Q A$ in which no classical negations and no unitary actions $a$ occur is equivalent to a formula of $Q L$ (and vice-versa).

Our above-mentioned Representation Theorem can be used to show that the translation of $Q L$ into $L Q A$ is actually faithful on arbitrary Quantum Transition Systems; more precisely, if for any QTS $\Sigma$ we denote by $\imath: \Sigma \hookrightarrow \Sigma(H)$ its canonical embedding into (the lattice of closed linear subspaces associated to) an infinite-dimensional Hilbert space $H$, then we have, for all states $s$ and all formulas $\varphi$ of $Q L$ :

$$
s \models_{l(\Sigma)} \varphi \text { iff } s \models \Sigma \varphi^{t r} .
$$

As a consequence of these faithfulness results, $L Q A$ is a conservative extension of $Q L$; i.e. for all formulas $\varphi$ of $Q L$, we have: $\models \varphi$ iff $\models \varphi^{t r}$.

\footnotetext{
7 The quantum dual $\sim[\varphi$ ?] $\sim \psi$ of the weakest precondition captures the (strongest) effect induced by (a given cause) $\psi$ after a test $\varphi$ ? is performed. This corresponds to what is known in CS as the strongest post-condition that is actualized by performing an action $\varphi$ ? on a state satisfying (the precondition) $\psi$.
} 


\section{Conclusions: quantum "non-classicality" is dynamical}

In this paper we argued that the "non-classicality" of Quantum Logic is only due to the non-classical "dynamics" of quantum information, and thus it does not require any change of the classical laws governing "static" information. While accepting the challenge posed by the "empirical" view of Logic, we nevertheless found no empirically grounds within Quantum Mechanics to reject Classical Logic.

Let us recall (from Sect.3) the first two points in the currently most widely-held view on Quantum Logic, according to the excellent overview in Bacciagaluppi (2008): (a) quantum logic is suitable as a logic that locally replaces classical logic when used to describe "a class of propositions in the context of quantum mechanical experiments"; (b) it is still open whether or not quantum logic does globally replace classical logic.

Our answer to (a) is that classical logic is, or at least, can be claimed to be (without any contradiction with Quantum Mechanics) the logic of all static propositions. Of course, if one arbitrarily restricts the family of properties to only some of them (the "testable" ones), then it might happen that this restricted class does not naturally form a Boolean algebra. However, as we pointed out in the examples given in Sect. 3, nothing shows that the "testable" properties are the only physically meaningful ones. Instead, what is physically interesting about "testable" properties is that they can be directly "tested", i.e. one can design a "test" (a measurement) which applied to any system yields the "yes" answer if and only if after the test the system satisfies the given property. Also, it yields the "yes" answer with probability 1 if and only if before the test the system satisfied the given property.

Hence in the view we now put forward, the non-classical character of the "logic" of quantum-testable properties is not due to the fact that they are properties of a quantum system, but to the fact that we required them to be "testable" by quantum measurements. It is the non-classical nature of quantum actions (in particular, quantum tests) that explains the strangeness of quantum behavior. This has to do with the system's behavior under interaction: a test is performed, some answer is obtained, and this establishes a "testable" property. It has nothing to do with the validity or non-validity of the laws of classical propositional logic: those laws were not even meant to talk about interactions or dynamics, but they were laws governing "static" information about systems at one fixed moment in time. The laws of testable properties are thus non-classical simply because they talk about something else than classical logic: they talk about the effects of potential changes. This answer forms the basis of our Quantum Dynamic Logic, and gives a principled explanation for the "nonclassicality" of Quantum Logic. Classical logical laws are unapplicable to quantumlogical operators simply because these are not static-propositional operators! One can still concede that, in a sense, Quantum Logic "locally replaces" classical logic, but this happens only when attempting to describe in a quasi-propositional manner what in fact are dynamic properties governing quantum interactions. But the classical logical connectives are essentially static in nature: they are simply ways to combine and relate "static" propositions talking about a given system in the absence of any interaction. If one treats the quantum-logical operators appropriately (in terms of axioms about dynamic modalities), then quantum-logical laws can safely coexist within the same 
logical calculus with the laws of classical propositional logic: indeed, our logic $L Q A$ provides precisely such a common setting.

The point (b) is even more controversial: as explained in Bacciagaluppi (2008), it deals with the question whether quantum logic is the one and only "true logic" and if so it asks for the reasons why its connectives should replace the classical ones. Both answers "yes" and "no" are taken to be plausible answers for (b) in Bacciagaluppi (2008), where the arguments are influenced by the specific interpretation of quantum mechanics that one accepts. But our principled explanation to point (a) suggests that (b) is something of a red herring. We can see absolutely no empirical or conceptual reason why quantum logical connectives should "globally replace" the classical ones. As we saw, even when they "locally" do, they can in fact happily live with the classical ones together in the same calculus.

Our formalism is enlightening, because it dispels some of the mysticism surrounding Quantum Mechanics: there is no need to "evade” Classical Logic. All that Quantum Mechanics shows (and Quantum Dynamic Logic formalizes) is the fact that certain informational interactions change the systems involved; and moreover, they change them non-locally. (But this last aspect can only be formalized in the multi-partite version of our Quantum Dynamic Logic, see Baltag and Smets 2004, 2006a, 2008, 2010.) As a dynamic logic, quantum logic may still be in some sense "non-classical": its tests do not behave like classical PDL tests, since they change the state. We see no problem with this. That is to say, we have no strong a priori logical intuitions that "tests" or other physical actions must behave "classically" in some (rather obscure) sense. The specific effects of a physical action are a matter of experimentation and not of a priori reasoning. However, we do have prior logical intuitions about negation and disjunction. We accept that these intuitions may be in principle be challenged by physical reality. But if we have to choose between accepting that measurements can sometimes change the state of a measured system or giving up all our intuitions about propositional connectives, the choice is easy! Our stance on this clearly goes against Putnam's 1968 views in (Putnam 1968), where he states that "to stipulate that certain sentences shall be immune from revision is irrational if that stipulation may lead one into serious difficulties, such as having to postulate either mysterious disturbances by the measurement (or to say that the measurement brings what it measures into existence) or "hidden variables"” (Putnam 1968, p. 196).

We started by taking seriously Putnam's (and Beth's) empirical conception of Logic. In this spirit, we addressed the challenge posed by Quantum Logic by tracking back the original experimental-operational meaning of quantum-logical concepts, following the lead of the operationalist tradition of the Geneva school. We arrived (on the footprints of the Brussel school of quantum logic) to the conclusion that the true meaning of these concepts is dynamic. In the spirit of Beth and van Fraassen, we proceeded to give a formal semantics to quantum logic, based on this dynamic interpretation. We obtained a Quantum Dynamic Logic, very much in the spirit of the "dynamic turn" in logic promoted by the Dutch school. Our formal results allow us now to go back and address critically Putnam's views about the inescapable necessity to give up the laws of classical logic. Instead of being "irrational", the explanations based on "mysterious disturbances by the measurement" are seen to be correct, clear and providing a clean formal setting for quantum logic. In contrast, Putnam's proposed "escape 
from Logic" seems in the light of our formal semantical analysis very similar to the "pseudo-scientific tendencies" evoked by Beth: one of the false "lessons" that philosophers too often tend to "learn" in a hurry from empirical sciences. In other words, our semantic work can be claimed to have done exactly what Beth was expecting it to do!

Open Access This article is distributed under the terms of the Creative Commons Attribution Noncommercial License which permits any noncommercial use, distribution, and reproduction in any medium, provided the original author(s) and source are credited.

\section{References}

Amira, H., Coecke, B., \& Stubbe, I. (1998). How quantales emerge by introducing induction within the operational approach. Helvetica Physica Acta, 71, 554-572.

Bacciagaluppi, G. (2008). Is logic empirical? In K. Engesser, D. M. Gabbay, \& D. Lehmann (Eds.), Handbook of quantum logic and quantum structures. Amsterdam: Elsevier.

Baltag, A., \& Smets, S. (2004). The logic of quantum programs. In P. Selinger (Ed.), Proceedings of the second international workshop on quantum programming languages (QPL2004), TUCS General Publication (Vol. 33, pp. 39-56). Turku Center for Computer Science.

Baltag, A., \& Smets, S. (2005). Complete axiomatizations of quantum actions. International Journal of Theoretical Physics, 44(12), 2267-2282.

Baltag, A., \& Smets, S. (2006a). LQP: The dynamic logic of quantum information. In Mathematical structures in computer science, Special Issue on Quantum Programming Languages Vol. 16(3), pp. 491-525.

Baltag, A., \& Smets, S. (2006b). Logics for quantum information flow. Lecture notes, Course presented at ESSLLI2006, Malaga, Spain. www.vub.ac.be/CLWF/SS/slides.html.

Baltag, A., \& Smets, S. (2008). A dynamic-logical perspective on quantum behavior. In L. Horsten \& I. Douven (Eds.), Special issue: Applied logic in the philosophy of science. Studia logica. 89, $178-211$.

Baltag, A., \& Smets, S. (2010). Correlated knowledge, an epistemic-logic view on quantum entanglement. International Journal of Theoretical Physics. Accessed 1 July 2010.

Bell, J., \& Hallett, M. (1982). Logic, quantum logic and empiricism. Philosophy of Science, 49, 355-379.

Beth, E. W. (1948). De wetenschap als cultuurfactor. In De functie der wetenschap. Tweede symposion der Sociëteit voor Culturele Samenwerking, s Gravenhage, 7-19, 1948. Translated from Dutch as "Science as a cultural factor (1948)" in E. W. Beth, Science a road to wisdom, collected philosophical studies, D. Reidel Publishing Company, Dordrecht, 1968.

Beth, E. W. (1948/49). Analyse sémantique des théories physiques. Synthese, 7, 206-207.

Beth, E. W. (1949a). Natuurphilosophie. Gorinchem: Noorduijn en zoon n.v.

Beth, E. W. (1949b). Towards an up-to-date philosophy of the natural sciences. Methodos, 1, 178-185.

Beth, E. W. (1960). Semantics of physical theories. Synthese, 12, 172-175.

Beth, E. W. (1968a). Scientific philosophy: Its aims and means (1948). In Science a road to wisdom, collected philosophical studies. Dordrecht: D. Reidel Publishing Company.

Beth, E. W. (1968b). "In Retrospect (1960)", translated from Dutch in Science a road to wisdom, collected philosophical studies. Dordrecht: D. Reidel Publishing Company.

Birkhoff, G., \& von Neumann, J. (1936). The logic of quantum mechanics. Annals of Mathematics, 37, 823-843, reprinted in C. A. Hooker (Ed.), The Logico-algebraic approach to quantum mechanics (Vol. 1, pp. 1-26). Dordrecht: D. Reidel Publishing Company.

Butrick, R. (1971). Discussion: Putnam's revolution. Philosophy of Science, 38, 290-292.

Cattaneo, C., \& Nisticó, G. (1991). Axiomatic foundations of quantum physics: Critiques and misunderstandings. Piron's question-proposition system. International Journal of Theoretical Physics, 30, 1293-1336.

Coecke, B., Moore, D. J., \& Smets, S. (2004). Logic of dynamics \& dynamics of logic; some paradigm examples. In S. Rahman, J. Symons, D.M. Gabbay, \& J. P. Van Bendegem (Eds.), Logic, epistemology and the unity of science (pp. 527-556). Netherlands: Springer. 
Coecke, B., Moore, D. J., \& Stubbe, I. (2001). Quantaloids describing causation and propagation for physical properties. Foundations of Physics Letters, 14, 357-367.

Coecke, B., \& Smets, S. (2004). The Sasaki hook is not a [static] implicative connective but induces a backward [in time] dynamic one that assigns causes. International Journal of Theoretical Physics, 43, 1705-1736.

Dalla Chiara, M., Giuntini, R., \& Greechie, R. (2004). Reasoning in quantum theory, sharp and unsharp quantum logics. Dordrecht: Kluwer Academic Publishers.

Daniel, W. (1989). Axiomatic description of irreversible and reversible Evolution of a physical system. Helvetica Physica Acta, 62, 941-968.

Drieschner, M. (1977). Is (quantum) logic empirical? Journal of Philosophical Logic, 6, 415-423.

Dummett, M. (1976). Is logic empirical? In H. D. Lewis (Ed.), Contemporary British Philosophy (pp. 45-68). London: George Allen and Unwin. Reprinted in M. Dummett, Truth and Other Enigmas, p.269-289, Duckwort UK, 1978

Faure, C. L.-A., Moore, D. J., \& Piron, C. (1995). Deterministic evolutions and Schrodinger flows. Helvetica Physica Acta, 68, 150-157.

Friedman, M., \& Glymour, C. (1972). If quanta had logic. Journal of Philosophical Logic, 1, 16-28.

Gardner, M. R. (1971). Is Quantum logic really logic? Philosophy of Science, 38, 508-529.

Goldblatt, R. (1974). Semantic analysis of orthologic. Journal of Philosophical Logic, 3, 19-35.

Goldblatt, R. (1984). Orthomodularity is not elementary. Journal of Symbolic Logic, 49, 401-404.

Harel, D., Kozen, D., \& Tiuryn, J. (2000). Dynamic logic. Cambridge: MIT Press.

Hughes, R. I. G. (1979). Realism and quantum logic. In G. E. Beltrametti \& B. C. van Fraassen (Eds.), Current issues in quantum logic (pp. 77-87). New York: Plenum Press.

Jauch, J. M. (1968). Foundations of quantum mechanics, reading. Reading, MA: Addison-Wesley.

Jauch, J. M., \& Piron, C. (1969). On the structure of quantal proposition systems. Helvetica Physica Acta, 42, 842-848.

Jauch, J. M., \& Piron, C. (1970). What is "Quantum-Logic"? In P. G. O. Freund, C. J. Goebel, \& Y. Nambu (Eds.), Quanta. Chicago: University Press.

Mayet, R. (1998). Some characterizations of the underlying division ring of a hilbert lattice by automorphisms. International Journal of Theoretical Physics, 37(1), 109-114.

Moore, D. J. (1999). On state spaces and property lattices. Studies in History and Philosophy of Modern Physics, 30, 61-83.

Piron, C. (1964). Axiomatique quantique (Ph.D. thesis). Helvetica Physica Acta, 37, 439-468. English Translation by M. Cole: Quantum Axiomatics RB4 Technical memo 107/106/104, GPO Engineering Department, London.

Piron, C. (1976). Foundations of quantum physics. Reading, MA: W.A. Benjamin Inc.

Piron, C. (1990). Mécanique quantique.Bases et applications (1st ed.). Lausanne: Presses polytechniques et universitaires romandes (Second corrected edition 1998).

Putnam, H. (1968). Is logic empirical? In R. Cohen \& M. Wartofsky (Eds.), Boston studies in the philosophy of science (Vol. 5). Dordrecht: D. Reidel Publishing Company.

Smets, S. (2001a). On causation and a counterfactual in quantum logic: The Sasaki hook. Logique et Analyse, 173-175, 307-325.

Smets, S. (2001b). The logic of physical properties, in static and dynamic perspective. Ph.D. thesis, Free University of Brussels.

Smets, S. (2006). From intuitionistic logic to dynamic operational quantum logic. Poznan Studies in Philosophy and the Humanities, 91, 257-275.

Solèr, M. P. (1995). Characterization of Hilbert spaces by orthomodular spaces. Communications in Algebra, 23(1), 219-243.

Stairs, A. (1983). Quantum logic, realism, and value definiteness. Philosophy of Science, 50, 578-602.

van Benthem, J. (1996). Exploring logical dynamics, studies in logic, language and information. Stanford: CSLI Publications.

van Benthem, J. (in press). Logical dynamics of information and interaction. Cambridge University Press, to appear.

van Benthem, J., van Ulsen, P., \& Visser, H. (Eds.). (2008). Logic and scientific philosophy. An E.W. Beth centenary celebration. Amsterdam: Beth Foundation.

van Fraassen, B. C. (1970). On the extension of Beth's semantics of physical theories. Philosophy of Science, 37(3), 325-339. 
van Fraassen, B. C. (1987). The semantic approach to scientific theories. In N. J. Nersessian (Ed.), The process of science: Contemporary philosophical approaches to understanding scientific practice (pp. 104-124). Dordrecht: Kluwer Academic Publishers.

von Neumann, J. (1932). Grundlagen der Quantenmechanik. Berlin: Springer Verlag. English translation Mathematical Foundations of Quantum Mechanics. New Jersey: Princeton University Press, 1996. 